\title{
Simply connected K-contact and Sasakian manifolds of dimension 7
}

\author{
Vicente Muñoz $^{1,2} \cdot$ Aleksy Tralle $^{3}$
}

Received: 13 August 2014 / Accepted: 12 May 2015 / Published online: 31 July 2015

(C) The Author(s) 2015. This article is published with open access at Springerlink.com

\begin{abstract}
We construct a compact simply connected 7-dimensional manifold admitting a $\mathrm{K}$-contact structure but not a Sasakian structure. We also study rational homotopy properties of such manifolds, proving in particular that a simply connected 7-dimensional Sasakian manifold has vanishing cup product $\mathrm{H}^{2} \times \mathrm{H}^{2} \rightarrow \mathrm{H}^{4}$ and that it is formal if and only if all its triple Massey products vanish.
\end{abstract}

Keywords Sasakian manifold · Contact structure $\cdot$ Symplectic manifold · Formality

Mathematics Subject Classification $\quad 53 \mathrm{C} 25 \cdot 53 \mathrm{D} 35 \cdot 57 \mathrm{R} 17 \cdot 55 \mathrm{P} 62$

\section{Introduction}

Sasakian geometry has become an important and active subject, especially after the appearance of the fundamental treatise of Boyer and Galicki [3]. Chapter 7 of this book contains an extended discussion of the topological problems in the theory of Sasakian, and, more generally, K-contact manifolds. These are odd-dimensional analogues to Kähler and symplectic manifolds, respectively.

\section{Aleksy Tralle}

tralle@matman.uwm.edu.pl

Vicente Muñoz

vicente.munoz@mat.ucm.es

1 Facultad de Ciencias Matemáticas, Universidad Complutense de Madrid, Plaza de Ciencias 3, 28040 Madrid, Spain

2 Instituto de Ciencias Matemáticas (CSIC-UAM-UC3M-UCM), C/Nicolás Cabrera 15, 28049 Madrid, Spain

3 Department of Mathematics and Computer Science, University of Warmia and Mazury, Słoneczna 54, 10-710 Olsztyn, Poland 
The precise definition is as follows. Let $(M, \eta)$ be a co-oriented contact manifold with a contact form $\eta \in \Omega^{1}(M)$, that is $\eta \wedge(d \eta)^{n}>0$ everywhere, with $\operatorname{dim} M=2 n+1$. We say that $(M, \eta)$ is $K$-contact if there is an endomorphism $\Phi$ of $T M$ such that:

- $\Phi^{2}=-\mathrm{Id}+\xi \otimes \eta$, where $\xi$ is the Reeb vector field of $\eta$ (that is $i_{\xi} \eta=1, i_{\xi}(d \eta)=0$ ),

- the contact form $\eta$ is compatible with $\Phi$ in the sense that $d \eta(\Phi X, \Phi Y)=d \eta(X, Y)$, for all vector fields $X, Y$,

- $d \eta(\Phi X, X)>0$ for all nonzero $X \in \operatorname{ker} \eta$, and

- the Reeb field $\xi$ is Killing with respect to the Riemannian metric defined by the formula $g(X, Y)=d \eta(\Phi X, Y)+\eta(X) \eta(Y)$.

In other words, the endomorphism $\Phi$ defines a complex structure on $\mathcal{D}=\operatorname{ker} \eta$ compatible with $d \eta$, hence $\Phi$ is orthogonal with respect to the metric $\left.g\right|_{\mathcal{D}}$. By definition, the Reeb vector field $\xi$ is orthogonal to $\operatorname{ker} \eta$, and it is a Killing vector field.

Let $(M, \eta, g, \Phi)$ be a K-contact manifold. Consider the contact cone as the Riemannian manifold $C(M)=\left(M \times \mathbb{R}^{>0}, t^{2} g+d t^{2}\right)$. One defines the almost complex structure $I$ on $C(M)$ by:

- $I(X)=\Phi(X)$ on ker $\eta$,

- $I(\xi)=t \frac{\partial}{\partial t}, I\left(t \frac{\partial}{\partial t}\right)=-\xi$, for the Killing vector field $\xi$ of $\eta$.

We say that $(M, \eta, \Phi, g, I)$ is Sasakian if $I$ is integrable. Thus, by definition, any Sasakian manifold is $\mathrm{K}$-contact.

There are several topological obstructions to the existence of the aforementioned structures on a compact manifold $M$ of dimension $2 n+1$, for example:

(1) the evenness of the $p$ th Betti number for $p$ odd with $1 \leq p \leq n$, of a Sasakian manifold,

(2) some torsion obstructions in dimension 5 discovered by Kollár [17],

(3) the fundamental group of Sasakian manifolds are special,

(4) the cohomology algebra of a Sasakian manifold satisfies the hard Lefschetz property,

(5) formality properties of the rational homotopy type.

An early result [13] establishes that the odd Betti numbers up to the middle dimension of Sasakian manifolds must be even. The parity of $b_{1}$ was used to produce the first examples of K-contact manifolds with no Sasakian structure [3, example 7.4.16]. More refined tools are needed in the case of even Betti numbers. The cohomology algebra of a Sasakian manifold satisfies a hard Lefschetz property [4]. Using it examples of K-contact non-Sasakian manifolds are produced in [5] in dimensions 5 and 7. These examples are nilmanifolds with even Betti numbers, so in particular they are not simply connected.

The fundamental group can also be used to construct K-contact non-Sasakian manifolds. Fundamental groups of Sasakian manifolds are called Sasaki groups, and satisfy strong restrictions. Using this it is possible to construct (non-simply connected) compact manifolds which are K-contact but not Sasakian [8].

When one moves to the case of simply connected manifolds, K-contact non-Sasakian examples of any dimension $\geq 9$ were constructed in [16] using the evenness of the third Betti number of a compact Sasakian manifold. Alternatively, using the hard Lefschetz property for Sasakian manifolds there are examples [19] of simply connected K-contact non-Sasakian manifolds of any dimension $\geq 9$.

In [24] and in [2] the rational homotopy type of Sasakian manifolds is studied. In [2] it is proved that all higher order Massey products for simply connected Sasakian manifolds vanish, although there are Sasakian manifolds with non-vanishing triple Massey products. This yields examples of simply connected K-contact non-Sasakian manifolds in dimensions $\geq 17$. However, Massey products are not suitable for the analysis of lower dimensional manifolds. 
Hence, the problem of the existence of simply connected K-contact non-Sasakian compact manifolds (open problem 7.4.1 in [3]) is still open in dimensions 5 and 7. Dimension 5 is the most difficult one, and it is treated in [3] separately. Here one has to use the obstructions of [17] which are very subtle torsion obstructions associated to the classification of Kähler surfaces. By definition, a simply connected compact oriented 5-manifold is called a SmaleBarden manifold. These manifolds are classified topologically by $H_{2}(M, \mathbb{Z})$ and the second Stiefel-Whitney class. Chapter 10 of the book by Boyer and Galicki is devoted to a description of some Smale-Barden manifolds which carry Sasakian structures. The following problem is still open (open problem 10.2.1 in [3]).

Do there exist Smale-Barden manifolds which carry K-contact but do not carry Sasakian structures?

In this note we solve the described problem in the easier case of dimension 7 (the solution is still possible by means of homotopy theory combined with symplectic surgery).

Theorem 1 There exist 7-dimensional compact simply connected $K$-contact manifolds which do not admit a Sasakian structure.

We then turn around to the study of the rational homotopy type of K-contact and Sasakian simply connected manifolds of dimension 7 . In particular, we prove:

Corollary 2 Let $M$ be a simply connected compact $K$-contact 7-dimensional manifold. Suppose that the cup product map $H^{2}(M) \times H^{2}(M) \longrightarrow H^{4}(M)$ is non-zero. Then $M$ does not admit a Sasakian structure.

Formality is a very useful rational homotopy property that has been widely used to distinguish between symplectic and Kähler manifolds [21] (see Sect. 6 for definitions and details). Simply connected compact manifolds of dimension $\leq 6$ are always formal, so formality becomes interesting in dimension 7 . We study this property in detail giving a precise characterisation for Sasakian manifolds (see Theorem 15). In particular, we have the following:

Corollary 3 Let $M$ be a simply connected compact Sasakian 7-dimensional manifold. Then $M$ is formal if and only if all triple Massey products are zero.

\section{Gompf-Cavalcanti manifold}

Let $(M, \omega)$ be a symplectic manifold of dimension $2 n$. For every $0 \leq k \leq n$, we define the Lefschetz map as $L_{\omega}: H^{n-k}(M) \rightarrow H^{n+k}(M), L_{\omega}([\beta])=\left[\beta \wedge \omega^{n-k}\right]$. We say that $M$ satisfies the hard Lefschetz property if $L_{\omega}$ is an isomorphism for every $0 \leq k \leq n$.

Proposition 4 There exists a simply connected 6-dimensional symplectic manifold $(M, \omega)$ such that $\operatorname{dim} \operatorname{ker}\left(L_{\omega}: H^{2}(M) \rightarrow H^{4}(M)\right)$ is odd.

Proof Gompf constructs in [14, Theorem 7.1] an example of a simply connected 6dimensional symplectic manifold $(M, \omega)$ which does not satisfy the hard Lefschetz property, that is, the Lefschetz map $L_{\omega}: H^{2}(M) \rightarrow H^{4}(M)$ is not an isomorphism. If $\operatorname{dim} \operatorname{ker} L_{\omega}$ is already odd then we have finished.

So let us suppose that $\operatorname{dim} \operatorname{ker} L_{\omega}$ is even. Take a cohomology class $a \in H^{2}(M)$ which belongs to the kernel of $L_{\omega}$. In [7, Lemma 2.4] Cavalcanti proves that given a symplectic manifold $(M, \omega)$ as above satisfying that there exists a symplectic surface $S \hookrightarrow M$ with 
$\langle a,[S]\rangle \neq 0$, then there is another 6-dimensional symplectic manifold $\left(M^{\prime}, \omega^{\prime}\right)$ (the symplectic blow-up of $M$ along $S$ ) satisfying

$$
\operatorname{dim} \operatorname{ker}\left(L_{\omega^{\prime}}: H^{2}\left(M^{\prime}\right) \rightarrow H^{2}\left(M^{\prime}\right)\right)=\operatorname{dim} \operatorname{ker}\left(L_{\omega}: H^{2}(M) \rightarrow H^{2}(M)\right)-1 .
$$

The symplectic blow-up of $M$ along $S$ is constructed in [20], where it is proved that the fundamental groups $\pi_{1}\left(M^{\prime}\right) \cong \pi_{1}(M)$, hence $M^{\prime}$ is simply connected. This means that the simply connected 6-dimensional symplectic manifold $M^{\prime}$ satisfies that $\operatorname{dim} \operatorname{ker}\left(L_{\omega^{\prime}}\right.$ : $\left.H^{2}\left(M^{\prime}\right) \rightarrow H^{4}\left(M^{\prime}\right)\right)$ is odd, as required.

It remains to find $S \hookrightarrow M$ as required. The cohomology class $a$ is non-zero, so there is some $b \in H^{4}(M, \mathbb{Z})$ such that $a \cup b \neq 0$. It is easy to see that there is a rank 2 complex vector bundle $E \rightarrow M$ with $c_{1}(E)=0, c_{2}(E)=2 b$. This corresponds to the fact that the map $[M, B \mathrm{SU}(2)] \rightarrow H^{4}(M, \mathbb{Z})$ given by the second Chern class exhausts $2 H^{4}(M, \mathbb{Z})$. A short proof runs as follows: $B \mathrm{SU}(2)$ has trivial 3-skeleton and it has $\pi_{4}(B \mathrm{SU}(2))=\mathbb{Z}$ and $\pi_{5}(B \mathrm{SU}(2))=\mathbb{Z}_{2}$. Represent the cohomology class $b$ by a cocycle $\varphi_{b}: C_{4}(M) \rightarrow \mathbb{Z}$, where $C_{4}(M)$ is the space of cellular chains. Given $b$, we define $f: M \rightarrow B$ SU(2) inductively on the skeleta (in what follows we denote by $X[k]$ the $k$-skeleton of a space $X$ ). It is trivial on the 3-skeleton of $M$. For every 4-cell $c$, we define $f: c \rightarrow B \mathrm{SU}(2)[4]=S^{4}$ to have degree $\varphi_{b}(c) \in \mathbb{Z}$. As $M$ is simply connected there are no 5-cells, so it only remains to attach the 6-cell $c_{6}$ to the 4-skeleton $M$ [4]. The attaching map is given by some $g: S^{5} \rightarrow M[4]$. When composed with $f$, we have a map $f \circ g: S^{5} \rightarrow B$ SU(2), which gives an obstruction element $o_{f} \in \pi_{5}(B \mathrm{SU}(2))=\mathbb{Z}_{2}$. If we multiply $b$ by two, then the map $\varphi_{b}$ gets multiplied by 2 . The corresponding $f$ is given by composing $f$ with a double cover of $S^{4}$, hence the obstruction element is $2 o_{f}=0$. This means that the map $f$ associated to $2 b$ can be extended to $M \rightarrow B \mathrm{SU}(2)$.

Now take the rank 2 bundle $E \rightarrow M$ just constructed. Assume that $[\omega]$ is a an integral cohomology class (which can always be done by perturbing $\omega$ slightly to make it rational and multiplying it by a large integer). Let $L \rightarrow M$ be the line bundle with first Chern class $c_{1}(L)=[\omega]$. We now use the asymptotically holomorphic techniques introduced by Donaldson [10]. Specifically, the result of [1] guarantees the existence of a suitable large $k \gg 0$ and a section of $E \otimes L^{\otimes k}$ whose zero locus is a symplectic manifold (an asymptotically holomorphic manifold in fact). This zero locus $S \subset M$ is a symplectic surface, and the cohomology class defined by $S$ is $c_{2}\left(E \otimes L^{\otimes k}\right)=c_{2}(E)+2 k c_{1}(L)=2 b+2 k[\omega]$. Therefore $\langle a,[S]\rangle=\langle a, 2 b+2 k[\omega]\rangle=2\langle a, b\rangle \neq 0$, as required.

We will call the manifold produced in Proposition 4 the Gompf-Cavalcanti manifold, because it is constructed by the surgery technique of Gompf [14] together with the symplectic blow-up of Cavalcanti [7]. Note however that this is not a unique one but a family of manifolds.

\section{Simply-connected K-contact non-Sasakian manifolds in dimension 7}

We show the existence of simply connected compact K-contact non-Sasakian manifolds in dimension 7 by proving that the Boothby-Wang fibration over the Gompf-Cavalcanti manifold is K-contact but non-Sasakian. The existence of a K-contact structure on such fibration is shown in [2] and [16]. For the convenience of the reader we briefly recall these constructions.

Let $(B, \omega)$ be a symplectic manifold such that the cohomology class $[\omega]$ is integral. Consider the principal $S^{1}$-bundle $\pi: M \rightarrow B$ given by the cohomology class $[\omega] \in$ $H^{2}(B, \mathbb{Z})$. Fibrations of this kind were first considered by Boothby and Wang and are called 
Boothby-Wang fibrations. By [25], the total space $M$ carries an $S^{1}$-invariant contact form $\eta$ such that $\eta$ is a connection form whose curvature is $d \eta=\pi^{*} \omega$. We have the following result (which is known, compare Theorem 6.1.26 and Proposition 7.1.2 in [3]).

Theorem 5 Any Boothby-Wang fibration admits a K-contact structure on the total space.

Proof To prove this theorem we need to introduce a certain tool, called the universal contact moment map in the sense of Lerman [18]. Recall that by our assumption the given contact distribution $\mathcal{D}$ is determined by the contact form $\eta$, that is $\mathcal{D}=\operatorname{ker} \eta$. Consider its annihilator $\mathcal{D}^{0} \subset T^{*} M$. Clearly, $\mathcal{D}^{0}$ is a line bundle, and, therefore, it has two components after the removal of the zero section,

$$
\mathcal{D}^{0} \backslash M=\mathcal{D}_{+}^{0} \sqcup \mathcal{D}_{-}^{0} .
$$

Single out one of these components, say $\mathcal{D}_{+}^{0}$. Consider the Lie algebra of contact vector fields $\chi(M, \eta)$ on $M$. It is known that this Lie algebra can be identified with a space of sections of the vector bundle $T M / \mathcal{D}$, that is $\chi(M, \eta) \cong \Gamma(M, T M / \mathcal{D})$. Because of that there is a natural pairing between points of the line bundle $\mathcal{D}^{0}$ and contact vector fields given by the formula

$$
\mathcal{D}^{0} \times \chi(M, \eta) \rightarrow \mathbb{R}, \quad((p, \beta), X) \mapsto\left\langle\beta, X_{p}\right\rangle
$$

where $\beta \in \mathcal{D}^{0}, X_{p} \in T_{p} M, p \in M$. Suppose that a Lie algebra $\mathfrak{g}$ acts on $M$ by contact vector fields, that is, there exists a representation $\rho: \mathfrak{g} \rightarrow \chi(M, \eta)$. Define the universal moment map as the map

$$
\psi: \mathcal{D}_{+}^{0} \rightarrow \mathfrak{g}^{*}
$$

by the formula

$$
\langle\psi(p, \beta), X\rangle=\langle(p, \beta), \rho(X)\rangle=\left\langle\beta, \rho(X)_{p}\right\rangle,
$$

where $(p, \beta) \in\left(\mathcal{D}_{+}^{0}\right)_{p} \subset T_{p}^{*} M, X \in \mathfrak{g}$. Now the proof becomes a consequence of the following criterion proved by Lerman [18].

Proposition 6 A compact co-orientable contact manifold $(M, \eta)$ admits a $K$-contact metric $g$ if and only if there exists an action of a torus $T$ on $M$ preserving the contact structure $\mathcal{D}$ and a vector $X \in \mathfrak{t}=L(T)$ so that the function $\langle\psi, X\rangle: \mathcal{D}_{+}^{0} \rightarrow \mathbb{R}$ is strictly positive.

We continue with the proof of Theorem 5. Consider the $S^{1}$-action on $M$ given by the Reeb vector field. Let $\mathfrak{g}=L\left(S^{1}\right)$, and $\rho: \mathfrak{g} \rightarrow \chi(M, \eta)$ be the homomorphism of Lie algebras determined by this action (thus, $\mathfrak{g}=\mathfrak{t}=L\left(S^{1}\right)$ in this particular situation). Since the $S^{1}$-action is free, $\rho(X)_{p} \neq 0$ for any $p \in M$. Now,

$$
\langle\psi, X\rangle(p, \beta)=\langle\psi(p, \beta), X\rangle=\left\langle\beta, \rho(X)_{p}\right\rangle .
$$

Note that in the considered case $\beta \in\left(\mathcal{D}_{+}^{0}\right)_{p} \subset T_{p}^{*} M$, and, therefore, $\beta \neq 0$. Also $(p, \beta)$ belongs to the annihilator of the distribution $\mathcal{D}$, while $\rho(X)$ is transversal to $\mathcal{D}$, since it is given by the Reeb vector field. Thus, for any point $p,\left\langle(p, \beta), \rho(X)_{p}\right\rangle \neq 0$. Hence, $X$ may be chosen to yield positive sign everywhere, and we complete the proof by applying Proposition 6.

Remark 7 Proposition 7.1.2 from [3] is due to Rukimbira. In this work we give a different proof based on Lerman's criterion given by Proposition 6 .

The following gives a proof of Theorem 1 . 
Theorem 8 The total space of the Boothby-Wang fibration over the Gompf-Cavalcanti manifold is a simply connected $K$-contact non-Sasakian manifold of dimension 7.

Proof Let $(M, \omega)$ be a Gompf-Cavalcanti manifold as given by Proposition 4 . We can assume that $[\omega]$ is an integral cohomology class. Let

$$
S^{1} \rightarrow E \rightarrow M
$$

be the associated Boothby-Wang fibration. By Theorem 5, $E$ has a K-contact structure. Now we need to prove that $E$ cannot carry Sasakian structures.

There is an exact sequence

$$
H_{2}(M) \rightarrow H_{1}\left(S^{1}\right)=\mathbb{Z} \rightarrow H_{1}(E) \rightarrow 0
$$

from the Serre spectral sequence. The map $H_{2}(M) \rightarrow \mathbb{Z}$ is cupping with $[\omega] \in H^{2}(M)$. Taking $[\omega]$ integral cohomology class and primitive, we have that $H_{2}(M) \rightarrow \mathbb{Z}$ is surjective and hence $H_{1}(E)=0$. The long homotopy exact sequence gives $\pi_{1}\left(S^{1}\right)=\mathbb{Z} \rightarrow \pi_{1}(E) \rightarrow$ $\pi_{1}(M)=0$, hence $\pi_{1}(E)$ is abelian. Therefore $E$ is simply connected.

The Gysin exact sequence associated to (1) is

$$
H^{1}(M)=0 \stackrel{\wedge \omega}{\longrightarrow} H^{3}(M) \longrightarrow H^{3}(E) \longrightarrow H^{2}(M) \stackrel{\wedge \omega}{\longrightarrow} H^{4}(M) .
$$

Thus

$$
b^{3}(E)=b^{3}(M)+\operatorname{dim}\left(\operatorname{ker} L_{\omega}: H^{2}(M) \rightarrow H^{4}(M)\right) .
$$

As $M$ is a 6-manifold, we have that $b^{3}(M)$ is even (by Poincaré duality, the intersection pairing on $H^{3}(M)$ is an antisymmetric non-degenerate bilinear form, hence the dimension of $H^{3}(M)$ is even). By construction, $\operatorname{dim}\left(\operatorname{ker} L_{\omega}: H^{2}(M) \rightarrow H^{4}(M)\right)$ is odd, so $b^{3}(E)$ is odd. As the third Betti number of a 7-dimensional Sasakian manifold has to be even [13], we have that $E$ cannot admit a Sasakian structure.

\section{Regularity and quasi-regularity}

A Sasakian or a K-contact structure on a compact manifold $M$ is called quasi-regular if there is a positive integer $\delta$ satisfying the condition that each point of $M$ has a foliated coordinate chart $(U, t)$ with respect to $\xi$ (the coordinate $t$ is in the direction of $\xi$ ) such that each leaf for $\xi$ passes through $U$ at most $\delta$ times. If $\delta=1$, then the Sasakian or K-contact structure is called regular (see [3, p. 188]).

If $N$ is a Kähler manifold whose Kähler form $\omega$ defines an integral cohomology class, then the total space of the circle bundle $S^{1} \hookrightarrow M \stackrel{\pi}{\longrightarrow} N$ with Euler class $[\omega] \in H^{2}(M, \mathbb{Z})$ is a regular Sasakian manifold with contact form $\eta$ such that $d \eta=\pi^{*}(\omega)$. The converse also holds: if $M$ is a regular Sasakian structure then the space of leaves $N$ is a Kähler manifold, and we have a circle bundle $S^{1} \rightarrow M \rightarrow N$ as above. If $M$ has a quasi-regular Sasakian structure, then the space of leaves $N$ is a Kähler orbifold with cyclic quotient singularities, and there is an orbifold circle bundle $S^{1} \rightarrow M \rightarrow N$ such that the contact form $\eta$ satisfies $d \eta=\pi^{*}(\omega)$, where $\omega$ is the orbifold Kähler form.

Similar properties hold in the K-contact case, substituting Kähler by symplectic (actually almost Kähler). If $M$ has a regular K-contact structure, then it is the total space of a circle bundle $S^{1} \hookrightarrow M \stackrel{\pi}{\longrightarrow} N$, where $(N, \omega)$ is a symplectic manifold, with Euler class $[\omega] \in$ $H^{2}(M, \mathbb{Z})$ and $d \eta=\pi^{*}(\omega)$. If $M$ has a quasi-regular K-contact structure, then it is the total 
space of an orbifold circle bundle $S^{1} \rightarrow M \rightarrow N$ over a symplectic orbifold $N$ with cyclic quotient singularities and Euler class $[\omega] \in H^{2}(M, \mathbb{Z})$, where $\omega$ is the orbifold symplectic form.

A result of [22] says that if $M$ admits a Sasakian structure, then it admits also a quasi-regular Sasakian structure. This also extends to the case of K-contact structures (Rukimbira [23], see also Theorem 7.1.10 in [3]).

Proposition 9 If a compact manifold $M$ admits a $K$-contact structure, it admits a quasiregular contact structure.

Proof Assume that there is a K-contact structure on $M$. By Proposition 6, there exists a torus action $T \times M \rightarrow M$ preserving the contact distribution and a vector $X \in \mathfrak{t}$ such that $\langle\psi, X\rangle>0$. Choose a vector $Y \in \mathfrak{t}$ with the property that it is tangent to an embedding $T^{\prime}=S^{1} \hookrightarrow T$. Clearly, the corresponding fundamental vector field $Y_{M}$ has the property that the leaves of the corresponding foliations are compact. The set of such $Y$ is dense in $t$. Therefore, for vectors $Y$ which are sufficiently close to $X$, the condition $\langle\psi, Y\rangle>0$ is still satisfied.

So it remains to see that there is K-contact structure whose Reeb vector field is $Y_{M}$, since this will be quasi-regular because the leaves of the characteristic foliation are all compact. We follow the notations of the proof of Theorem 5. The action of the circle $T^{\prime}$ on $M$ preserves $\mathcal{D}$, hence the lifted action of $T^{\prime}$ on $T^{*} M$ preserves $\mathcal{D}^{0}$. Since $T^{\prime}$ is connected, the lifted action preserves the connected component $\mathcal{D}_{+}^{0}$ as well. It follows that for any 1-form $\beta$ on $M$ with $\operatorname{ker} \beta=\mathcal{D}$, the average $\bar{\beta}$ of $\beta$ over $T^{\prime}$ still satisfies $\operatorname{ker} \bar{\beta}=\mathcal{D}$. So $\bar{\beta} \in \mathcal{D}^{0}$. Now use the formula [derived in [18], formulae (3.4) and (3.5)],

$$
i_{Y_{M}} \bar{\beta}=\langle\psi \circ \bar{\beta}, Y\rangle>0 \text {. }
$$

Now let

$$
\eta=(\langle\psi \circ \bar{\beta}, Y\rangle)^{-1} \bar{\beta},
$$

which satisfies $i_{Y_{M}} \eta=1$. Hence $\eta$ defines the contact structure and $Y_{M}$ is its Killing vector field. Then $T M=\mathcal{D} \oplus\left\langle Y_{M}\right\rangle$, and the splitting is $T^{\prime}$-invariant. We use the splitting to define the desired Riemannian metric $g$. Declare $\mathcal{D}$ and $\left\langle Y_{M}\right\rangle$ to be orthogonal and define $g\left(Y_{M}, Y_{M}\right)=1$, thus $Y_{M}$ becomes a unit normal to $\mathcal{D}$. On $\mathcal{D}$ we choose a $T^{\prime}$-invariant complex structure compatible with $\left.d \eta\right|_{\mathcal{D}}$ and define $\left.g\right|_{\mathcal{D}}(\cdot, \cdot)=\left.d \eta\right|_{\mathcal{D}}(\cdot, \Phi \cdot)$. Then $g$ is $T^{\prime}$-invariant and hence $L_{Y_{M}} g=0$. Thus we have obtained a K-contact structure on $M$.

\section{Minimal models and formality}

Now we want to analyse the rational homotopy type of K-contact and Sasakian simply connected 7-manifolds, in particular the property of formality. Simply connected compact manifolds of dimension $\leq 6$ are always formal [12], so dimension 7 is the first instance in which formality is an issue.

We start by reviewing concepts about minimal models and formality from [11,12,15]. A differential graded algebra (or DGA) over the real numbers $\mathbb{R}$, is a pair $(A, d)$ consisting of a graded commutative algebra $A=\oplus_{k \geq 0} A^{k}$ over $\mathbb{R}$, and a differential $d$ satisfying the Leibnitz rule $d(a \cdot b)=(d a) \cdot b+(-1)^{|a|} a \cdot(d b)$, where $|a|$ is the degree of $a$. Given a differential graded commutative algebra $(A, d)$, we denote its cohomology by $H^{*}(A)$. The cohomology of a differential graded algebra $H^{*}(A)$ is naturally a DGA with the product 
inherited from that on $A$ and with the differential being identically zero. The DGA $(A, d)$ is connected if $H^{0}(A)=\mathbb{R}$, and $A$ is 1-connected if, in addition, $H^{1}(A)=0$. Henceforth we shall assume that all our DGAs are connected. In our context, the main example of DGA is the de Rham complex $\left(\Omega^{*}(M), d\right)$ of a connected differentiable manifold $M$, where $d$ is the exterior differential.

Morphisms between DGAs are required to preserve the degree and to commute with the differential. A morphism $f:(A, d) \rightarrow(B, d)$ is a quasi-isomorphism if the map induced in cohomology $f^{*}: H^{*}(A, d) \rightarrow H^{*}(B, d)$ is an isomorphism. Quasi-isomorphism produces an equivalence relation in the category of DGAs.

\section{$\operatorname{A~DGA~}(\mathcal{M}, d)$ is minimal if}

(1) $\mathcal{M}$ is free as an algebra, that is, $\mathcal{M}$ is the free algebra $\bigwedge V$ over a graded vector space $V=\bigoplus_{i} V^{i}$, and

(2) there is a collection of generators $\left\{x_{\tau}\right\}_{\tau \in I}$ indexed by some well ordered set $I$, such that $\left|x_{\mu}\right| \leq\left|x_{\tau}\right|$ if $\mu<\tau$ and each $d x_{\tau}$ is expressed in terms of preceding $x_{\mu}, \mu<\tau$.

We say that $(\bigwedge V, d)$ is a minimal model of the differential graded commutative algebra $(A, d)$ if $(\bigwedge V, d)$ is minimal and there exists a quasi-isomorphism $\rho:(\bigwedge V, d) \longrightarrow$ $(A, d)$. A connected DGA $(A, d)$ has a minimal model unique up to isomorphism. For 1connected DGAs, this is proved in [9]. In this case, the minimal model satisfies that $V^{1}=0$ and the condition (2) above is equivalent to $d x_{\tau}$ not having a linear part.

A minimal model of a connected differentiable manifold $M$ is a minimal model $(\bigwedge V, d)$ for the de Rham complex $\left(\Omega^{*}(M), d\right)$ of differential forms on $M$. If $M$ is a simply connected manifold, then the dual of the real homotopy vector space $\pi_{i}(M) \otimes \mathbb{R}$ is isomorphic to $V^{i}$ for any $i$ (see [9]).

A model of a DGA $(A, d)$ is any DGA $(B, d)$ with the same minimal model (that is, they are equivalent with respect to the equivalence relation determined by the quasi-isomorphisms).

A minimal algebra $(\bigwedge V, d)$ is called formal if there exists a morphism of differential algebras $\psi:(\bigwedge V, d) \longrightarrow\left(H^{*}(\bigwedge V), 0\right)$ inducing the identity map on cohomology. Also a differentiable manifold $M$ is called formal if its minimal model is formal. The formality of a minimal algebra is characterized as follows.

Proposition 10 [9] A minimal algebra $(\bigwedge V, d)$ is formal if and only if the space $V$ can be decomposed into a direct sum $V=C \oplus N$ with $d(C)=0$ and $d$ injective on $N$, such that every closed element in the ideal $I(N)$ in $\bigwedge V$ generated by $N$ is exact.

This characterization of formality can be weakened using the concept of $s$-formality introduced in [12].

Definition 11 A minimal algebra $(\bigwedge V, d)$ is $s$-formal $(s>0)$ if for each $i \leq s$ the space $V^{i}$ of generators of degree $i$ decomposes as a direct sum $V^{i}=C^{i} \oplus N^{i}$, where the spaces $C^{i}$ and $N^{i}$ satisfy the three following conditions:

(1) $d\left(C^{i}\right)=0$,

(2) the differential map $d: N^{i} \longrightarrow \bigwedge V$ is injective, and

(3) any closed element in the ideal $I_{s}=I\left(\bigoplus_{i \leq s} N^{i}\right)$, generated by the space $\bigoplus_{i \leq s} N^{i}$ in the free algebra $\bigwedge\left(\bigoplus_{i \leq s} V^{i}\right)$, is exact in $\bigwedge V$.

A differentiable manifold $M$ is $s$-formal if its minimal model is $s$-formal. Clearly, if $M$ is formal then $M$ is $s$-formal, for any $s>0$. The main result of [12] shows that sometimes the weaker condition of $s$-formality implies formality. 
Theorem 12 [12] Let $M$ be a connected and orientable compact manifold of dimension $2 n$ or $(2 n-1)$. Then $M$ is formal if and only if it is $(n-1)$-formal.

By Corollary 3.3 in [12] a simply connected compact manifold is always 2-formal. Therefore, Theorem 12 implies that any simply connected compact manifold of dimension not more than six is formal. For simply connected 7-dimensional compact manifolds, we have that $M$ is formal if and only if $M$ is 3-formal.

Theorem 12 also holds for compact connected orientable orbifolds, since the proof of [12] only uses that the cohomology $H^{*}(M)$ is a Poincaré duality algebra.

\section{Homotopy properties of simply connected Sasakian 7-manifolds}

Proposition 13 Let $M$ be a simply connected compact $K$-contact 7-dimensional manifold. Then a model for $M$ is $(H \otimes \wedge(x), d)$, where $H$ is the cohomology algebra of a simply connected symplectic 6-dimensional orbifold and $d x=\omega \in H^{2}$ is the class of the symplectic form.

If $M$ is Sasakian, then $H$ is the cohomology algebra of a simply connected 6-dimensional Kähler orbifold.

Proof Suppose $M$ admits a Sasakian structure. Then $M$ admits a quasi-regular Sasakian structure [22]. Therefore, there is an orbifold circle bundle $S^{1} \rightarrow M \rightarrow B$, where $B$ is a compact Kähler orbifold of dimension 6, with Euler class given by the Kähler form $\omega \in$ $H^{2}(B)$. We note that $B$ is simply connected because $M$ is so (see [3, Theorem 4.3.18]). In particular, $S^{1} \rightarrow M \rightarrow B$ is a rational fibration, hence if $\mathcal{M}$ is a model for $B$, then $\mathcal{M} \otimes \bigwedge(x)$, with $|x|=1, d x=\omega$, is a model for $M$.

Now $B$ is a simply connected compact orbifold of dimension 6 . So it is 2-formal. Theorem 12 also holds for orbifolds, hence $B$ is formal. Therefore $\mathcal{M} \sim(H, 0)$, where $H=H^{*}(B)$ is the cohomology algebra of $B$. So a model for $M$ is of the form $(H \otimes \bigwedge(x), d)$, $d x=\omega \in H^{2}$.

The case where $M$ admits a K-contact structure is similar. By Proposition 9, it admits a quasi-regular K-contact structure. Therefore, $M$ is an orbifold $S^{1}$-bundle over a symplectic orbifold $S^{1} \rightarrow M \rightarrow B$, with Euler class given by the orbifold symplectic form $\omega \in H^{2}(B)$. As above, a model for $M$ is $(H \otimes \bigwedge(x), d), d x=\omega \in H^{2}$, where $H=H^{*}(B)$.

We prove now Corollary 2.

Corollary 14 Let $M$ be a simply connected compact $K$-contact 7-dimensional manifold. Suppose that the cup product map $H^{2}(M) \times H^{2}(M) \longrightarrow H^{4}(M)$ is non-zero. Then $M$ does not admit a Sasakian structure.

Proof Let us compute the cohomology of $M$ from its model $(\mathcal{M}, d)=(H \otimes \wedge(x), d)$, $d x=\omega$, where $H=H^{*}(B)$ is the cohomology algebra of a 6-dimensional simply connected symplectic manifold. Note that $\omega \in H^{2}$ is a non-zero element with $\omega^{3} \in H^{6}$ generating the top cohomology.

Consider the Lefschetz map $L_{\omega}: H^{*} \rightarrow H^{*+2}$, and let $K^{*}=\operatorname{ker} L_{\omega}, Q^{*}=\operatorname{coker} L_{\omega}$. We have a (non-canonical) isomorphism $H^{i}(M) \cong Q^{i} \oplus K^{i-1} x$. Note that $Q^{3}=K^{3}=H^{3}$ and $H^{6}=\mathbb{R}$. Also $Q^{2}=H^{2} /\langle\omega\rangle$, and $K^{4}=\operatorname{ker}\left(L_{\omega}: H^{4} \rightarrow \mathbb{R}\right)$ are vector spaces of codimension one. We have the following: 


$$
\begin{aligned}
& H^{0}(M)=\mathbb{R}, \\
& H^{1}(M)=0, \\
& H^{2}(M)=Q^{2}, \\
& H^{3}(M)=H^{3} \oplus K^{2} x, \\
& H^{4}(M)=Q^{4} \oplus H^{3} x, \\
& H^{5}(M)=K^{4} x, \\
& H^{6}(M)=0, \\
& H^{7}(M)=\left\langle\omega^{3} x\right\rangle .
\end{aligned}
$$

The map $H^{2}(M) \times H^{2}(M) \rightarrow H^{4}(M)$ factors through $Q^{2} \times Q^{2} \rightarrow Q^{4}$. Hence if it is nonzero then $Q^{4} \neq 0$. In particular, the Lefschetz map $L_{\omega}: H^{2} \rightarrow H^{4}$ is not an isomorphism, so $B$ is not hard Lefschetz.

If $M$ admits a Sasakian structure, then there is a quasi-regular fibration $S^{1} \rightarrow M \rightarrow B$ with $B$ satisfying the hard Lefschetz property (it is a Kähler orbifold, so [26] is applicable). This contradicts the above.

Now we shall study the case of Sasakian 7-manifolds in more detail. Let $M$ be a simply connected compact Sasakian 7-dimensional manifold. Then

$$
\mathcal{M}=(H \otimes \bigwedge(x), d)
$$

is a model for $M$, by Proposition 13, where $H=H^{*}(B)$ is the cohomology algebra of a simply connected compact 6-dimensional Kähler orbifold. This algebra $H$ has a very rich structure:

(1) there is a canonical isomorphism $H^{6} \cong \mathbb{R}$, which is given by integration $\int_{M}: H^{6} \rightarrow \mathbb{R}$;

(2) $H$ is a Poincaré duality algebra, hence $H^{3} \otimes H^{3} \rightarrow \mathbb{R}$ is an antisymmetric bilinear pairing;

(3) there is a scalar product on each $H^{j}$. This is given by the Hodge star operator $*: H^{j} \rightarrow$ $H^{6-j}$ combined with wedge and integration;

(4) $H$ has a Hodge structure, that is, $H \otimes \mathbb{C}$ has a bigrading such that $H^{k} \otimes \mathbb{C}=\oplus_{p+q=k} H^{p, q}$, where $H^{p, q}=\overline{H^{q, p}}$, and the wedge product respects the bigrading;

(5) there is a distinguished element $\omega \in H^{2}$ which is in $H^{1,1}$. This defines the space of primitive forms $P=\langle\omega\rangle^{\perp} \subset H^{2}$. Hence $H^{2}=\langle\omega\rangle \oplus P$. Moreover $P=P^{1,1} \oplus P^{2,0}$, where $P^{1,1}=P \cap H^{1,1}$ and $P^{2,0}=P \cap\left(H^{2,0} \oplus H^{0,2}\right)$;

(6) the Lefschetz map $L_{\omega}: H^{2} \rightarrow H^{4}$ is an isomorphism. Therefore $H^{4}=\left\langle\omega^{2}\right\rangle \oplus \omega P^{1,1} \oplus$ $\omega P^{2,0}$. By Theorem 3.16 of Chapter V of [27], for $\alpha_{1} \in P^{1,1}$ we have $* \alpha_{1}=-\alpha_{1} \wedge \omega$, for $\alpha_{2} \in P^{2,0}$ we have $* \alpha_{2}=\alpha_{2} \wedge \omega$, and $* \omega=\frac{1}{2} \omega^{2}$. This implies that $L_{\omega}:\langle\omega\rangle \oplus$ $P^{1,1} \oplus P^{2,0} \rightarrow\left\langle\omega^{2}\right\rangle \oplus \omega P^{1,1} \oplus \omega P^{2,0}$ is of the form $L_{\omega}(\alpha)=L_{\omega}\left(\alpha_{0}+\alpha_{1}+\alpha_{2}\right)=$ $\frac{1}{2} * \alpha_{0}-* \alpha_{1}+* \alpha_{2}$, where $\alpha=\alpha_{0}+\alpha_{1}+\alpha_{2}$ is the decomposition according to $H^{2}=\langle\omega\rangle \oplus P^{1,1} \oplus P^{2,0}$.

The Lefschetz map $L_{\omega}: H^{2} \rightarrow H^{4}$ is an isomorphism so there is an inverse $L_{\omega}^{-1}: H^{4} \rightarrow$ $H^{2}$. Using it, we can define a map $\mathcal{F}: P \times P \times P \times P \rightarrow \mathbb{R}$ by

$$
\mathcal{F}(\alpha, \beta, \gamma, \delta)=\int_{M} L_{\omega}^{-1}(\alpha \wedge \beta) \wedge \gamma \wedge \delta
$$


This clearly factors through $\mathrm{Sym}^{2} P \times \mathrm{Sym}^{2} P$. Using (5) above, we have the alternative description

$$
\mathcal{F}(\alpha, \beta, \gamma, \delta)=2\left\langle(\alpha \wedge \beta)_{0},(\gamma \wedge \delta)_{0}\right\rangle-\left\langle(\alpha \wedge \beta)_{1},(\gamma \wedge \delta)_{1}\right\rangle+\left\langle(\alpha \wedge \beta)_{2},(\gamma \wedge \delta)_{2}\right\rangle,
$$

from where it follows that $\mathcal{F}$ factors as a map $\operatorname{Sym}^{2}\left(\operatorname{Sym}^{2} P\right) \rightarrow \mathbb{R}$.

Let $\mathcal{K}_{M}$ be the kernel of the map $\operatorname{Sym}^{2}\left(\operatorname{Sym}^{2} P\right) \rightarrow \operatorname{Sym}^{4} P$. Then we define a map

$$
\mathcal{F}_{M}=\left.\mathcal{F}\right|_{\mathcal{K}_{M}}: \mathcal{K}_{M} \rightarrow \mathbb{R} .
$$

We have the following result.

Theorem 15 Let $M$ be a simply connected compact Sasakian 7-dimensional manifold. Then $M$ is formal if and only if $\mathcal{F}_{M}=0$.

Proof Using Theorem 12, we only have to check whether $M$ is 3-formal. For this we have to construct the minimal model $\rho:(\bigwedge V, d) \rightarrow \mathcal{M}=(H \otimes \bigwedge(x), d)$ up to degree 3 . This is easy:

$$
\begin{aligned}
& V^{1}=0, \\
& V^{2}=P, \\
& V^{3}=H^{3} \oplus N^{3}, \quad \text { where } N^{3}=\operatorname{Sym}^{2} P,
\end{aligned}
$$

where the differential is given by $d=0$ on $P$ and $H^{3}$, and $d: N^{3} \rightarrow \bigwedge V^{2}$ is the isomorphism Sym $^{2} P \rightarrow \bigwedge^{2} P$. The map $\rho$ is given as follows. $\rho: V^{2}=P \rightarrow \mathcal{M}^{2}=H^{2}$ is defined as the obvious (inclusion) map, $\rho: H^{3} \rightarrow \mathcal{M}^{3}=H^{3} \oplus H^{2} x$ is the inclusion on the first summand, and $\rho: N^{3}=\operatorname{Sym}^{2} P \rightarrow \mathcal{M}^{3}=H^{3} \oplus H^{2} x$ is defined as $\rho(\alpha \cdot \beta)=L_{\omega}^{-1}(\alpha \wedge \beta) x$. Note that

$$
\begin{aligned}
& d(\rho(\alpha \cdot \beta))=L_{\omega}^{-1}(\alpha \wedge \beta) d x=L_{\omega}^{-1}(\alpha \wedge \beta) \omega \\
& \quad=\alpha \wedge \beta=\rho(\alpha) \wedge \rho(\beta)=\rho(\alpha \wedge \beta)=\rho(d(\alpha \cdot \beta)),
\end{aligned}
$$

so $\rho$ is a DGA map. Clearly it is a 3-equivalence (it induces an isomorphism on cohomology up to degree 3 and an inclusion on degree 4).

The space of closed elements is $C^{3}=H^{3}$. Now let us check when the elements $z \in I\left(N^{3}\right)$ with $d z=0$ satisfy $[\rho(z)]=0 \in H^{*}(M)$. The only cases to check is when $z$ has degree 5 or 7. If $z$ has degree 5 , then $[\rho(z)] \neq 0$ if and only if there exists some $\beta \in P,[\rho(\beta)] \in H^{2}(M)$, such that $[\rho(z)] \wedge[\rho(\beta)] \neq 0$, by Poincaré duality. Hence $[\rho(z \beta)] \neq 0$. This means that we can restrict to elements $z$ of degree 7 , that is $z \in N^{3} \cdot \bigwedge^{2} P$.

Let $z \in N^{3} \cdot \bigwedge^{2} P \cong \operatorname{Sym}^{2} P \times \operatorname{Sym}^{2} P$. Then the map $d: N^{3} \cdot \bigwedge^{2} P \rightarrow \bigwedge^{4} P$ coincides the full symmetrization map $\operatorname{Sym}^{2} P \times \operatorname{Sym}^{2} P \rightarrow \operatorname{Sym}^{4} P$. So

$$
Z=\left.\operatorname{ker} d\right|_{I\left(N^{3}\right)^{7}}=\mathcal{K}_{M} \oplus \operatorname{Ant}^{2}\left(\operatorname{Sym}^{2} P\right),
$$

where $\operatorname{Ant}^{2}(W)$ denotes the antisymmetric 2-power of a vector space $W$.

Now we have to study the map

$$
\rho: Z \rightarrow H^{7}(M)=H^{6} x,
$$

and see if this is non-zero. This is given (on the basis elements) by

$$
\rho((\alpha \cdot \beta) \cdot(\gamma \cdot \delta))=\left(L_{\omega}^{-1}(\alpha \wedge \beta) \wedge \gamma \wedge \delta\right) x,
$$

so $\mathcal{F}_{M}=\left.\rho\right|_{\mathcal{K}_{M}}$. Note that $\rho$ automatically vanishes on $\operatorname{Ant}^{2}\left(\operatorname{Sym}^{2} P\right)$, hence $M$ is formal if and only if $\rho$ vanishes on $\mathcal{K}_{M}$ if and only if $\mathcal{F}_{M}=0$. 
According to Theorem 12, to check non-formality we have to test the relevant property (2) on any splitting $V^{3}=C^{3}+N^{\prime 3}$. If we take another splitting $V^{3}=C^{3}+N^{\prime 3}$, then the projection $\pi: V^{3} \rightarrow N^{3}$ gives an isomorphism $\pi: N^{\prime 3} \rightarrow N^{3}$, and so an isomorphism $N^{\prime 3} \cdot \operatorname{Sym}^{2} P \cong N^{3} \cdot \operatorname{Sym}^{2} P$. Clearly, $d \circ \pi=d$ on $N^{\prime 3}$, so the spaces of cycles correspond $\mathcal{K}^{\prime} \cong \mathcal{K}$. On the other hand $H^{3} \cdot H^{2} \cdot H^{2}=0$, so the maps $\rho: \mathcal{K} \rightarrow H^{6} x$ and $\rho: \mathcal{K}^{\prime} \rightarrow H^{6} x$ also correspond. This means that the corresponding $\mathcal{F}$ and $\mathcal{F}^{\prime}$ coincide under the isomorphism $\mathcal{K} \cong \mathcal{K}^{\prime}$. This means that the choice of splitting is not relevant.

This result means that the formality or non-formality of $M$ only depends on the cohomology algebra $H$. Theorem 15 can be applied to the examples in Section 5.3 of [2]. For instance for $B=\mathbb{C} P^{1} \times \mathbb{C} P^{1} \times \mathbb{C} P^{1}$, we have a simply connected Sasakian 7-manifold which is non-formal (Theorem 12 of [2]). For $B=\mathbb{C} P^{3}$, we have obviously $P=0$ and hence $M$ is formal.

The element $\mathcal{F}_{M}$ of Theorem 15 is the principal Massey product defined by Crowley and Nordström [6] for simply connected compact 7-manifolds in general. The principal Massey product is the full obstruction to formality for simply connected compact 7-manifolds.

Now we deduce Corollary 3.

Corollary 16 Let $M$ be a simply connected compact Sasakian 7-dimensional manifold. Then $M$ is formal if and only if all triple Massey products are zero.

Proof Suppose that $\mathcal{F}_{M} \neq 0$. We choose an orthonormal basis for $H^{2}=\left\langle e_{0}, e_{1}, \ldots, e_{m}\right\rangle$, where $e_{0}=\frac{1}{\sqrt{3}} \omega, P^{1,1}=\left\langle e_{1}, \ldots, e_{s}\right\rangle, P^{2,0}=\left\langle e_{s+1}, \ldots, e_{m}\right\rangle$. The vector space $\mathcal{K}_{M}$ is generated by elements of the form

$$
a_{i j k l}=\left(e_{i} \cdot e_{j}\right) \cdot\left(e_{k} \cdot e_{l}\right)-\left(e_{k} \cdot e_{j}\right) \cdot\left(e_{i} \cdot e_{l}\right),
$$

for $0 \leq i, j, k, l \leq m$ (here, as usual, the dot product means symmetric product). Now define the numbers

$$
\lambda_{i j k}=\int_{M} e_{i} \wedge e_{j} \wedge e_{k} \in \mathbb{R}
$$

for $0 \leq i, j, k \leq m$. Note that these numbers are fully symmetric on $i, j, k$. Also $\lambda_{000}=\frac{2}{\sqrt{3}}$ and $\lambda_{i j 0}=\frac{1}{\sqrt{3}} \varepsilon_{i} \delta_{i j}$, for $(i, j) \neq(0,0)$, where $\varepsilon_{i}=-1$ for $1 \leq i \leq s$ and $\varepsilon_{i}=1$ for $s+1 \leq i \leq m$. Then

$$
L_{\omega}^{-1}\left(e_{i} \wedge e_{j}\right)=2 *\left(e_{i} \wedge e_{j}\right)_{0}-\left(e_{i} \wedge e_{j}\right)_{1}+\left(e_{i} \wedge e_{j}\right)_{2}=2 \lambda_{i j 0} e_{0}+\sum_{t>0} \varepsilon_{t} \lambda_{i j t} e_{t}
$$

So

$$
\mathcal{F}_{M}\left(\left(e_{i} \cdot e_{j}\right) \cdot\left(e_{k} \cdot e_{l}\right)\right)=2 \lambda_{i j 0} \lambda_{k l 0}+\sum_{t>0} \varepsilon_{t} \lambda_{i j t} \lambda_{k l t}
$$

Evaluating $\mathcal{F}_{M}$ on $a_{i j k l}$ gives a set of equations to determine the formality of $M . M$ is nonformal when there exists some $a_{i j k l}$ with $\mathcal{F}_{M}\left(a_{i j k l}\right) \neq 0$. By [6], we have that the triple Massey product $\left\langle e_{i}, e_{j}, e_{k}\right\rangle$ is a well-defined element of $H^{5}(M)$ and it satisfies

$$
\mathcal{F}_{M}\left(a_{i j k l}\right)=\left\langle e_{i}, e_{j}, e_{k}\right\rangle \cup e_{l} .
$$

So $\left\langle e_{i}, e_{j}, e_{k}\right\rangle \neq 0$, as required. 
This result is of relevance since it is not known if for general simply connected compact 7-dimensional manifolds there are obstructions to formality different from triple Bianchi-Massey tensor, as remarked in [6]. It is true that for higher dimensional manifolds, there are obstructions to formality even when all Massey products (triple and higher order) can be zero.

Acknowledgments We thank Johannes Nordström for several illuminating conversations and for providing us with a copy of [6], and G. Bazzoni and B. Cappelletti-Montano for pointing us some references. We are grateful to the referee for a careful reading of the manuscript. The first author was partially supported by Project MICINN (Spain) MTM2010-17389. The second author was partly supported by the ESF Research Networking Programme CAST (Contact and Symplectic Topology).

Open Access This article is distributed under the terms of the Creative Commons Attribution 4.0 International License (http://creativecommons.org/licenses/by/4.0/), which permits unrestricted use, distribution, and reproduction in any medium, provided you give appropriate credit to the original author(s) and the source, provide a link to the Creative Commons license, and indicate if changes were made.

\section{References}

1. Auroux, D.: Asymptotically holomorphic families of symplectic submanifolds. Geom. Funct. Anal. 7, 971-995 (1997)

2. Biswas, I., Fernández, M., Muñoz, V., Tralle, A.: On Formality of Orbifolds and Sasakian Manifolds. arXiv:1402.6861

3. Boyer, C., Galicki, K.: Sasakian Geometry. Oxford University Press, Oxford (2007)

4. Cappelletti-Montano, B., de Nicola, A., Yudin, I.: Hard Lefschetz Theorem for Sasakian Manifolds. arXiv:1306.2896

5. Cappelletti-Montano, B., de Nicola, A., Marrero, J.C., Yudin, I.: Examples of Compact K-Contact Manifolds with No Sasakian Metric. arXiv:1311.3270

6. Crowley, D., Nordström, J.: The rational homotopy type of (n-1)-connected (4n-1)-manifolds. arXiv: $1505.04184 \mathrm{v} 1$

7. Cavalcanti, G.: The Lefschetz property, formality and blowing up in symplectic geometry. Trans. Am. Math. Soc. 359, 333-348 (2007)

8. Chen, X.: On the fundamental groups of compact Sasakian manifolds. Math. Res. Lett. 20, 27-39 (2013)

9. Deligne, P., Griffiths, P., Morgan, J., Sullivan, D.: Real homotopy theory of Kähler manifolds. Invent. Math. 29, 245-274 (1975)

10. Donaldson, S.: Symplectic submanifolds and almost-complex geometry. J. Differ. Geom. 44, 666-705 (1996)

11. Felix, Y., Halperin, S., Thomas, J.-C.: Rational Homotopy Theory. Springer, Berlin (2002)

12. Fernández, M., Muñoz, V.: Formality of Donaldson submanifolds. Math. Z. 250, 149-175 (2005)

13. Fujitani, T.: Complex-valued differential forms on normal contact Riemannian manifolds. Töhoku Math. J. 2(18), 349-361 (1966)

14. Gompf, R.: A new construction of symplectic manifolds. Ann. Math. 142, 537-696 (1995)

15. Griffiths, P., Morgan, J.W.: Rational Homotopy Theory and Differential Forms. Progress in Math. 16, Birkhäuser, Basel (1981)

16. Hajduk, B., Tralle, A.: On simply connected compact K-contact non-Sasakian manifolds. J. Fixed Point Theory Appl. (2015). doi:10.1007/s11784-015-0210-y

17. Kollár, J.: Circle actions on simply connected 5-manifolds. Topology 45, 643-672 (2006)

18. Lerman, E.: Contact fiber bundles. J. Geom. Phys. 49, 52-66 (2004)

19. Lin, Y.:P Lefschetz Contact Manifolds and Odd Dimensional Symplectic Geometry. arXiv:1311.1431

20. McDuff, D.: Examples of simply-connected symplectic non-Kählerian manifolds. J. Diff. Geom. 20, 267-277 (1984)

21. Oprea, J., Tralle, A.: Symplectic Manifolds with No Kähler Structure. Lecture Notes in Math, vol. 1661. Springer, Berlin (1997)

22. Ornea, L., Verbitsky, M.: Sasakian structures on CR-manifolds. Geom. Dedic. 125, 159-173 (2007)

23. Rukimbira, P.: Chern-Hamilton's conjecture and K-contactness. Houston J. Math. 21, 709-718 (1995)

24. Tievsky, A.: Analogues of Kähler Geometry on Sasakian Manifolds, Ph.D. Thesis. MIT (2008)

25. Weinstein, A.: Fat bundles and symplectic manifolds. Adv. Math. 37, 239-250 (1980) 
26. Wang, Z., Zafran, D.: A remark on the hard Lefschetz theorem for orbifolds. Proc. Am. Math. Soc. 137, 2497-2501 (2009)

27. Wells, R.O.: Differential Analysis on Complex Manifolds, GTM 65, 2nd edn. Springer, Berlin (1979) 